\title{
On the Sum-Rate of MIMO Interference Channel
}

\author{
Harpreet S. Dhillon and R. Michael Buehrer \\ Mobile and Portable Radio Research Group (MPRG), \\ Wireless@ Virginia Tech, Bradley Department of Electrical and Computer Engineering, \\ Virginia Polytechnic Institute and State University, Blacksburg, VA 24061, USA. \\ E-mail: \{harpreet.dhillon, buehrer\}@vt.edu
}

\begin{abstract}
The problem of maximizing the sum-rate of a MIMO interference channel is investigated. Each receiver node is assumed to perform single user detection by treating interference from other users as Gaussian noise. It is assumed that all the users share a single frequency band and no pre-coding is performed over time. The sum-rate maximization in such a setup is a longstanding open problem due to its non-linear non-convex nature. The solution, to date, has only been approximated using the local optimization algorithms. In this paper, we couple the branch and bound strategy with the reformulation and linearization technique (BB/RLT) to develop a global optimization algorithm that finds a provably optimal solution. This problem is essentially an optimal power control problem over spatial channels and should not be confused with some recent developments such as Interference Alignment (IA) that typically require pre-coding over temporal, spectral or spatial dimensions. As a comparison with the state of the art, we compare the sum-rate achievable in the current system with the one predicted by IA and draw some interesting conclusions. It should be noted however, that even though the sum-rate achievable by IA can be predicted by assuming $N / 2$ degrees of freedom in an $N$-user interference channel, the feasibility of IA over a limited number of signaling dimensions is an open problem.
\end{abstract}

Index Terms-MIMO interference channel, optimal sum-rate, optimal power control, interference alignment, non-convex nonlinear programming.

\section{INTRODUCTION}

C HARACTERIZATION of the capacity region of an Interference Channel (IC) is one of the most interesting research problems in information theory and wireless communications. The performance of wireless users is coupled to each other due to mutual interference when they co-exist in the same area and transmit over same frequency channel. This coupling effect makes the analysis of the capacity region quite challenging. The study of such channels from an information theoretic perspective was initiated by Shannon [1]. Until recently, our understanding of ICs was limited and we were only able to characterize the capacity of a two user Gaussian IC in certain special cases, such as the very high interference case [2]-[6]. In an important recent development, the capacity region of a two-user Gaussian IC was approximated to within one-bit accuracy of the true capacity region [7]-[8]. The exact characterization of the capacity region of an $N$-user Gaussian IC is still an open problem. It should be noted that all the above mentioned works consider the possibility of interference cancelation by assuming that each receiver is capable of multiuser detection.
The capacity characterization problem of a general $N$-user IC was recently addressed by mapping it to the problem of characterizing the degrees of freedom of an IC [9]-[14]. A single-antenna IC is said to have $D$ degrees of freedom if its sum-rate $(S R)$ can be expressed as:

$$
S R=D \log (S N R)+o(\log (S N R)),
$$

where $\log (S N R)$ represents the capacity of an isolated singleantenna link, $o(\log (S N R))$ is a residual capacity term and $S N R$ is the signal-to-noise ratio [10], [11]. The most significant finding of these studies is that an $N$-user IC has $N / 2$ degrees of freedom per orthogonal frequency, time or spatial channel. This result is quite remarkable since it guarantees that an IC can achieve at least half the capacity of its nointerference counterpart for any number of interfering users. It has been further shown that these $N / 2$ degrees of freedom are achievable by Interference Alignment (IA) [10]. The basic idea of IA is to pre-code the transmit signal such that it aligns with the interference signals at all receivers where it acts as interference and is orthogonal to interference at the desired receiver. Several pre-coding methods are shown to achieve IA for single antenna ICs with time or frequency selectivity [12], [13]. $N$-user MIMO ICs are also shown to achieve $N / 2$ degrees of freedom when the channels have infinite selectivity [14]. However, it is difficult to find analytical solutions to the IA problems in general and even the feasibility of IA over a limited number of signaling dimensions is an open problem [15].

Another popular way of characterizing the capacity of ICs is to maximize the achievable sum-rate by treating interference from other users as Gaussian noise, which is considered optimal for low interference scenarios. This case is most applicable to the ICs where the receiver nodes lack information about the structure of the interference signals. In such channels, the receiver nodes are capable of doing only single user detection and hence the interference can not be canceled. The sumrate maximization problem for such an IC was formulated in [16] by assuming that all the transmitter (Tx) and receiver (Rx) nodes have multiple antennas. The solution to this nonconvex problem has, to date, only been approximated by local optimization techniques [17], [18]. Though these approaches can quickly find a locally optimal solution, they cannot guarantee the global optimum for non-convex problems. A global optimization algorithm was recently developed in [19] by coupling the branch and bound method with the reformulation and linearization technique (BB/RLT) to find the maximum 
sum-rate of a MIMO IC under the assumption that each user's transmit power is equally distributed over all the transmit antennas [20]. This assumption eases the mathematical formulation of the problem significantly, but unfortunately, the simplified results do not provide the maximum achievable sum-rate for a general MIMO IC.

In this paper, we extend the BB/RLT algorithm to solve the MIMO IC sum-rate maximization problem treating interference as Gaussian noise. As expected, the optimal results are better than those achievable by transmitting equal power over all the transmit antennas. We also make an interesting comparison between the optimal sum-rate achievable by the proposed power control technique with the one achievable when we assume that an $N$-user IC achieves $N / 2$ degrees of freedom per orthogonal time, frequency or spatial channel (referred henceforth as the IA bound). Denoting the capacity of an isolated MIMO link by $C_{M}$, the IA bound can be evaluated as $\frac{N}{2} C_{M}$. In this case, an IC achieves half the sum-rate of that of its no-interference counterpart. The comparison shows that the optimal power control performs better than the IA bound in the low- $S N R$ regime for reasonably high number of users. This is because the IA bound is highly suboptimal in the low $S N R$ regime (since the residual capacity term $o(\log (S N R)$ ) can not be neglected in (1) in low $S N R$ regime) [21]. As expected, the performance of IA bound gets better as compared to power control with the increase in $S N R$.

The remainder of the paper is organized as follows. The system model and the simulation layout are briefly explained in Section II. The sum-rate maximization problem of a MIMO IC is formulated in Section III. The proposed global optimization technique is discussed in Section IV. Section V deals with the numerical results and the paper is concluded in Section VI.

\section{SySTEM MODEL}

Assumptions: In this study, we assume an IC consisting of $N$ MIMO users. Each user has a Tx node comprising of $n_{t}$ antennas and a $\mathrm{Rx}$ node comprising of $n_{r}$ antennas. In this peer-peer network model each Tx has only one intended $\mathrm{Rx}$ and acts as an interferer for the rest of the users. An example of the basic system setup is shown in Fig. 1. We also assume that each $\mathrm{Rx}$ node has no information about the structure of the interference signals and hence decodes its intended signal by treating interference as Gaussian noise. Each Tx is assumed to have a total maximum transmit power of $P_{\max }$ over all $n_{t}$ transmit antennas. It is further assumed that all the users are sharing complete information and the system is centrally optimized to find the maximum sum of the mutual information. In this case, a centralized server obtains information about the topology (channel gains, etc.) and determines the optimal power allocation over the spatial channels for each user. We also assume that no temporal or spectral pre-coding is performed explicitly to achieve IA.

System Layout: The system layout is shown in Fig. 1. The maximum allowable distance between a Tx/Rx pair is denoted by $d_{\max } . d_{\max }$ is assumed to be a system constant and is defined such that the minimum average received SNR per receive antenna is $S N R_{\min } \mathrm{dB}$. The density of the users is handled by defining a Multi-User Interference (MUI) factor. $M U I$ represents the expected number of $\mathrm{Rx}$ nodes within a circle of radius $d_{\max }$ centered at any Tx node assuming a constant density. Increasing the $M U I$ factor increases the density of interferers and hence increases the mutual interference. For a fixed $M U I$ and $d_{\max }$ value, the density $(\mu)$ in terms of users per unit area can be evaluated as: $\mu=M U I / \pi d_{\text {max }}^{2}$. The complete area of interest is assumed to be a square. To place $N$ users within a square with density $\mu$, the square should have an area of $\frac{N}{\mu}$, and hence a side length of $\sqrt{\frac{N}{\mu}}$. For each analysis, we place a number of Rx units uniformly distributed in the chosen square area. Each Tx is then placed in the circle of radius $d_{\max }$ centered at the corresponding $\mathrm{Rx}$, as shown in Fig. 1. We simulate this system a number of times to find the ergodic sum-rate and for each simulation we place the users randomly as described above.

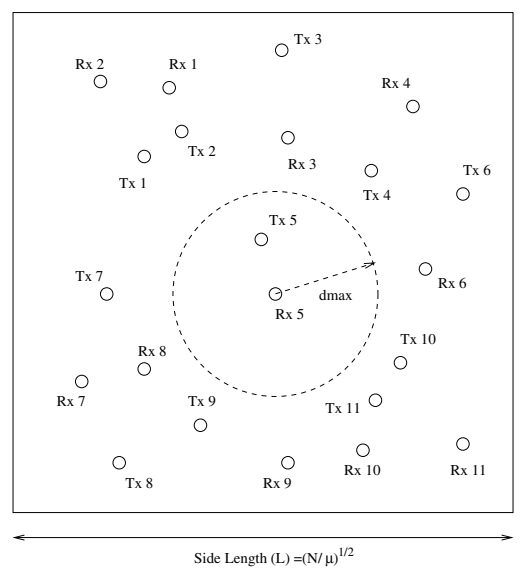

Fig. 1. System model depicting various mutually interfering Tx-Rx links.

Mathematical Notation: Boldface is used to denote matrices and vectors. For a matrix $\mathbf{A}, \mathbf{A}^{\dagger}$ denotes the conjugate transpose and $\operatorname{Tr}\{\mathbf{A}\}$ denotes the trace. I denotes the identity matrix, whose dimensions can be determined from the context. $\mathbf{A} \succ \mathbf{0}$ denotes that $\mathbf{A}$ is Hermitian and positive semidefinite. The scalar $a_{m n}$ represents the entry in the $m^{t h}$-row and $n^{t h}$ column of A. For a complex scalar $a, \Re(a)$ and $\Im(a)$ represent the real and imaginary parts of $a$ respectively, and $a^{\prime}$ represents the conjugate of $a \cdot \operatorname{diag}\{\mathbf{A}\} \succeq \mathbf{0}$ denotes that all the diagonal elements of $\mathbf{A}$ are non-negative.

\section{SuM-RATE OF MIMO INTERFERENCE CHANNEL}

Defining the Variables: Let us denote the MIMO link from the Tx of the $j^{t h}$ user to the Rx of the $i^{t h}$ user to be $L_{j i}$. Let $\mathbf{H}_{j i} \in C^{n_{r} \times n_{t}}$ denote the channel matrix of link $L_{j i}$. Let $\mathbf{Q}_{i}$ be the covariance matrix of the zero mean Gaussian input symbol vector $\mathbf{x}_{i}$ of the $i^{t h}$ user, i.e., $\mathbf{Q}_{i}=E\left\{\mathbf{x}_{i} \cdot \mathbf{x}_{i}^{\dagger}\right\}$. Further denote $\rho_{j i}$ as the signal-to-noise ratio if $j=i$, or the interference-to-noise ratio if $j \neq i$. Let $\mathbf{R}_{i}$ represent the covariance matrix of interference plus noise at the $\mathrm{Rx}$ node of the $i^{t h}$ user. Assuming interference plus noise to be Gaussian 
distributed, $\mathbf{R}_{i}$ can be computed as:

$$
\mathbf{R}_{i}=\sum_{\substack{j=1 \\ j \neq i}}^{N} \rho_{j i} \mathbf{H}_{j i} \mathbf{Q}_{j} \mathbf{H}_{j i}^{\dagger}+\mathbf{I} .
$$

Sum-Rate of MIMO Interference Channel: As discussed in [16], the information theoretic capacity of a single link in a MIMO IC can be computed as $C_{i}=\log _{2} \operatorname{det}(\mathbf{I}+$ $\left.\rho_{i i} \mathbf{R}_{i}^{-1} \mathbf{H}_{i i} \mathbf{Q}_{i} \mathbf{H}_{i i}^{\dagger}\right)$. Since $\mathbf{Q}_{i} \succ \mathbf{0}$, it can be expressed as $\mathbf{Q}_{i}=\mathbf{U}_{i} \Lambda_{i} \mathbf{U}_{i}^{\dagger}$, where $\Lambda_{i}$ is a diagonal matrix of the eigenvalues of $\mathbf{Q}_{i}$ and $\mathbf{U}_{i}$ is a unitary matrix with columns consisting of the eigenvectors of $\mathbf{Q}_{i}$. Defining $\widehat{\mathbf{H}}_{i i}=\mathbf{H}_{i i} \mathbf{U}_{i}$, the capacity expression can be written as $C_{i}=\log _{2} \operatorname{det}\left(\mathbf{I}+\rho_{i i} \mathbf{R}_{i}^{-1} \widehat{\mathbf{H}}_{i i} \Lambda_{i} \widehat{\mathbf{H}}_{i i}^{\dagger}\right)$. Since $\mathbf{Q}_{i} \succ \mathbf{0}$, it leads to the following two very important properties:

1) The distributions of $\widehat{\mathbf{H}}_{i i}$ and $\mathbf{H}_{i i}$ are same [16].

2) All the eigenvalues of $\mathbf{Q}_{i}$, i.e., all the diagonal elements of $\Lambda_{i}$, are real and positive.

Due to these properties, it is sufficient to optimize over $\Lambda_{i}, \forall i$, to find the optimal sum-rate. The mathematical formulation of the optimization problem is as follows:

$$
\begin{array}{ll}
\max & \sum_{i=1}^{N} C_{i} \\
\text { s.t. } & C_{i}=\log _{2} \operatorname{det}\left(\mathbf{R}_{i}+\rho_{i i} \widehat{\mathbf{H}}_{i i} \Lambda_{i} \widehat{\mathbf{H}}_{i i}^{\dagger}\right)-\log _{2} \operatorname{det}\left(\mathbf{R}_{i}\right) \\
& \mathbf{R}_{i}=\sum_{\substack{j=1 \\
j \neq i}}^{N} \rho_{j i} \widehat{\mathbf{H}}_{j i} \Lambda_{j} \widehat{\mathbf{H}}_{j i}^{\dagger}+\mathbf{I} \\
& \operatorname{Tr}\left\{\Lambda_{i}\right\} \leq P_{\max }, \operatorname{diag}\left\{\Lambda_{i}\right\} \succeq \mathbf{0}, 1 \leq i \leq N .
\end{array}
$$

\section{The Proposed MEthod}

Brief Overview of $B B / R L T$ : The basic idea of BB/RLT is to first find the upper bound (UB) and the lower bound (LB) to the optimal sum-rate by constructing a linear programming relaxation (LPR) of the original non-linear (NL) problem and then tightening the bounds by employing the $\mathrm{BB}$ algorithm [20]. While constructing the LPR, all of the NL terms in the objective function and the constraints are replaced by linear variables by introducing suitable linear constraints, thereby relaxing the feasible region. The optimal objective function value of this LP thus serves as an upper bound (UB) to the original NL problem (since it is a maximization problem). If the optimal solution of this relaxed LP is feasible to the original NL problem, it acts as a lower bound (LB) to the globally optimal value (since it is sub-optimal to the original NL problem in general). Otherwise, it can be used as a starting point to find a feasible solution by local search. After finding the UB and LB, the BB strategy is employed to partition the search space so that both the largest UB and LB approach the global optimum. When the largest UB and the LB are within some threshold, $\epsilon$, the BB/RLT is complete and yields a LB to the global optimum that is within $\epsilon$ of the true global optimum [20]. An illustration of the convergence of LB and UB to global optimum is shown in Fig. 2 for $\epsilon=10^{-4}$ and $N=3$. The BB/RLT algorithm as summarized in [19] is presented in Table I.

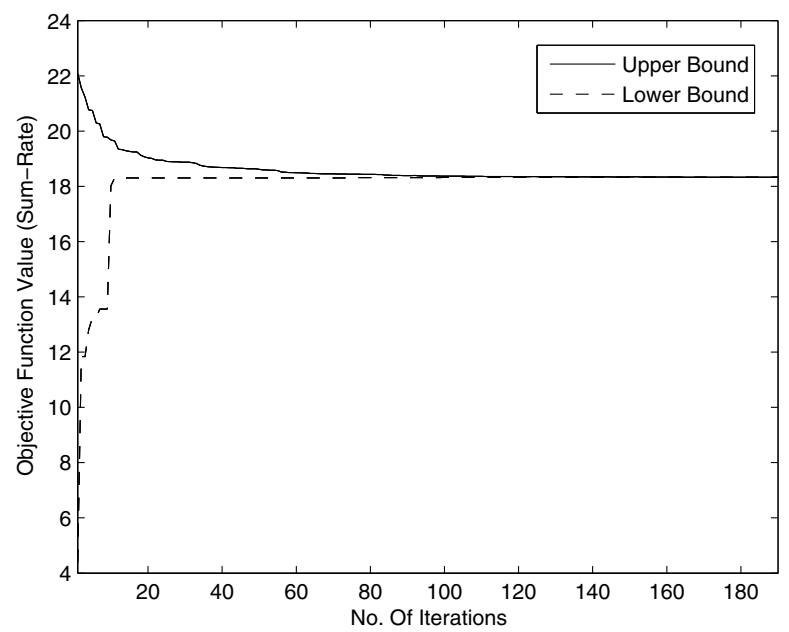

Fig. 2. Convergence of BB/RLT algorithm for a single instantiation of the interference channel $\left(\epsilon=10^{-4}, N=2\right.$ and $\left.M U I=1\right)$.

TABLE I

BB/RLT ALGORITHM

\section{Initialization:}

1. Let optimal solution $\alpha^{*}=\emptyset$. The initial $L B=-\infty$.

2 . Let the problem list contain only the original problem $\left(P_{1}\right)$.

3 . Introduce one new variable for each nonlinear term. Add linear constraints for these variables to build a linear relaxation.

4. Denote the solution to linear relaxation as $\hat{\alpha}$ and its objective value as upper bound $U B_{1}$.

Main Loop:

1. Select problem $P_{n}$ with the largest $U B$ from the problem list.

2. Find, if necessary, a feasible solution $\alpha_{n}$ via a local search algorithm for problem $P_{n}$. Denote the objective value by $L B_{n}$.

3. If $L B_{n}>L B$ then let $\alpha^{*}=\alpha_{n}$ and $L B=L B_{n}$. If $L B \geq(1-\epsilon) U B$ then stop with the $\epsilon$-optimal solution $\alpha^{*}$; else, remove all problems $P_{n^{\prime}}$ having $(1-\epsilon) U B_{n^{\prime}} \leq L B$ from the problem list.

4. Select a partitioning variable and divide its bounding interval equally into two new intervals.

5. Remove the selected problem $P_{n}$ from the problem list, construct two new problems $P_{n 1}$ and $P_{n 2}$ based on the two partitioned intervals.

6. Compute two new upper bounds $U B_{n 1}$ and $U B_{n 2}$ by solving the linear relaxations of $P_{n 1}$ and $P_{n 2}$, respectively.

7. If $(1-\epsilon) U B_{n 1} \geq L B$ then add problem $P_{n 1}$ to the problem list. Similarly, if $(1-\epsilon) U B_{n 2} \geq L B$ then add problem $P_{n 2}$ to the problem list.

8. If the problem list is empty, stop with the $\epsilon$-optimal solution $\alpha^{*}$. Otherwise, go to the Main Loop again.

Constructing an LPR: One of the important contributions of this paper is the LPR construction for the general MIMO IC sum-rate maximization problem. We begin by defining the following new variables to linearize the expression of $C_{i}$ :

$$
\begin{aligned}
& x_{i}=\operatorname{det}\left(\mathbf{R}_{i}+\rho_{i i} \widehat{\mathbf{H}}_{i i} \Lambda_{i} \widehat{\mathbf{H}}_{i i}^{\dagger}\right) ; u_{i}=\ln \left(x_{i}\right) ; \\
& y_{i}=\operatorname{det}\left(\mathbf{R}_{i}\right) ; v_{i}=\ln \left(y_{i}\right) .
\end{aligned}
$$

$C_{i}$ can be expressed in terms of the new variables as: $C_{i}=\frac{1}{\ln 2}\left(u_{i}-v_{i}\right)$. The constraints given by (4) are added to the problem formulation given by (3). $u_{i}$ and $v_{i}$ are logarithmic functions of the form $w=\ln z$, where $z \in\left[\begin{array}{ll}z_{L B} & z_{U B}\end{array}\right]$. Since, $x_{i}$ and $y_{i}$ are dependent upon $\Lambda_{i}$, their LB and UB can be 


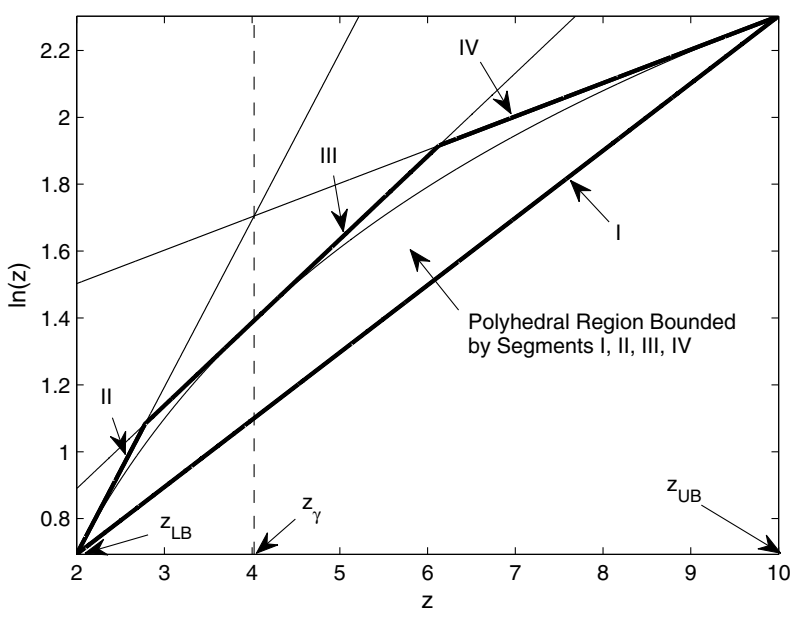

Fig. 3. Polyhedral approximation of $w=\ln z$.

calculated by evaluating their expressions at the LB and UB of $\Lambda_{i}$ respectively. A convex polygonal outer approximation is used to linearize the logarithmic function [19]. As shown in Fig. 3, the convex region is defined by the tangents at $\left(z_{L B}, \ln z_{L B}\right),\left(z_{\gamma}, \ln z_{\gamma}\right)$ and $\left(z_{U B}, \ln z_{U B}\right)$, and the chord joining $\left(z_{L B}, \ln z_{L B}\right)$ and $\left(z_{U B}, \ln z_{U B}\right)$, where $z_{\gamma}$ is the $\mathrm{z}$-coordinate of the point of intersection of the tangents at $\left(z_{L B}, \ln z_{L B}\right)$ and $\left(z_{U B}, \ln z_{U B}\right)$, which is given by:

$$
z_{\gamma}=\frac{z_{L B} z_{U B}\left(\ln z_{U B}-\ln z_{L B}\right)}{z_{U B}-z_{L B}} .
$$

The convex region defined by these four segments can be expressed by the following linear constraints:

$$
\begin{aligned}
& z_{L B} w-z \leq z_{L B}\left(\ln z_{L B}-1\right), \\
& z_{\gamma} w-z \leq z_{\gamma}\left(\ln z_{\gamma}-1\right), \\
& z_{U B} w-z \leq z_{U B}\left(\ln z_{U B}-1\right), \\
& \left(z_{U B}-z_{L B}\right) w+\left(\ln z_{L B}-\ln z_{U B}\right) z \geq \\
& z_{U B} \ln z_{L B}-z_{L B} \ln z_{U B} .
\end{aligned}
$$

After linearizing $u_{i}$ and $v_{i}$, we linearize $x_{i}$ and $y_{i}$. The expressions for $x_{i}$ and $y_{i}$ are quite similar and this similarity can be established by substituting $\mathbf{R}_{i}$ in (4):

$$
\begin{aligned}
& x_{i}=\operatorname{det}\left(\sum_{j=1}^{N}\left(\rho_{j i} \widehat{\mathbf{H}}_{j i} \Lambda_{j} \widehat{\mathbf{H}}_{j i}^{\dagger}+\mathbf{I}\right)\right) \triangleq \operatorname{det} \mathbf{S}_{i}, \\
& y_{i}=\operatorname{det}\left(\sum_{\substack{j=1 \\
j \neq i}}^{N}\left(\rho_{j i} \widehat{\mathbf{H}}_{j i} \Lambda_{j} \widehat{\mathbf{H}}_{j i}^{\dagger}+\mathbf{I}\right)\right) \triangleq \operatorname{det} \mathbf{R}_{i} .
\end{aligned}
$$

Keeping in mind this similarity, we illustrate the linearization process for only $x_{i}$, and the results for $y_{i}$ can be inferred directly. For the purpose of illustration, we assume that Tx and $\mathrm{Rx}$ nodes of each user have two antennas each. Since $\Lambda_{j}(\forall j)$ is an $n_{t} \times n_{t}(=2 \times 2)$ matrix, it can be expressed as:

$$
\Lambda_{j}=\left[\begin{array}{cc}
\lambda_{j 1} & 0 \\
0 & \lambda_{j 2}
\end{array}\right],
$$

where $\lambda_{j 1}$ and $\lambda_{j 2}$ are the fractions of power transmitted by the $j^{\text {th }}$ user over the two Eigen-modes of the MIMO channel. To simplify the expressions for $x_{i}$ and $y_{i}$ (given by (7)), let us define $\widetilde{\mathbf{H}}_{j i}=\sqrt{\rho_{j i}} \widehat{\mathbf{H}}_{j i}$. $\mathbf{S}_{i}$ can now be expressed as (9).
Taking the determinant of $\mathbf{S}_{i}, x_{i}$ can be expressed as (10). The expression for $y_{i}$ is similar to that for $x_{i}$ with the only difference being that the terms corresponding to $i^{t h}$ users are not included in the summations.

From (10) we observe that $x_{i}$ is a quadratic polynomial with three types of quadratic terms, viz., $\lambda_{j 1} \lambda_{k 1}, \lambda_{j 2} \lambda_{k 2}$ and $\lambda_{j 1} \lambda_{k 2}$. It is important to note that $\lambda_{j 1} \lambda_{k 1}$ is the same as $\lambda_{k 1} \lambda_{j 1}$ and hence the number of unique quadratic terms for this category is $\sum_{j=1}^{N} j=\frac{N}{2}(N+1)$. The same argument holds for $\lambda_{j 2} \lambda_{k 2}$. The number of unique terms for $\lambda_{j 1} \lambda_{k 2}$ $(\forall j, k)$ is $N^{2}$, which makes the total number of quadratic terms in (10) equal to $N(2 N+1)$. Each of these quadratic terms is replaced by a new variable: $\Gamma_{j 1 k 1}=\lambda_{j 1} \lambda_{k 1}, \Gamma_{j 1 k 2}=$ $\lambda_{j 1} \lambda_{k 2}$ and $\Gamma_{j 2 k 2}=\lambda_{j 2} \lambda_{k 2}$. These equalities are relaxed by including the following linear inequalities called bounding factor constraints (illustrated for $\Gamma_{j 1 k 2}$ ), for each non-linear term:

$$
\begin{aligned}
& \Gamma_{j 1 k 2}-\lambda_{j 1 L B} \lambda_{k 2}-\lambda_{j 1} \lambda_{k 2 L B} \geq-\lambda_{j 1 L B} \lambda_{k 2 L B} \\
& \Gamma_{j 1 k 2}-\lambda_{j 1 U B} \lambda_{k 2}-\lambda_{j 1} \lambda_{k 2 L B} \leq-\lambda_{j 1 U B} \lambda_{k 2 L B} \\
& \Gamma_{j 1 k 2}-\lambda_{j 1 L B} \lambda_{k 2}-\lambda_{j 1} \lambda_{k 2 U B} \leq-\lambda_{j 1 L B} \lambda_{k 2 U B} \\
& \Gamma_{j 1 k 2}-\lambda_{j 1 U B} \lambda_{k 2}-\lambda_{j 1} \lambda_{k 2 U B} \geq-\lambda_{j 1 U B} \lambda_{k 2 U B}
\end{aligned}
$$

This completes LPR construction of the original problem (3) and the corresponding LP can be expressed as:

$$
\begin{array}{ll}
\max & \sum_{i=1}^{N} C_{i} \\
\text { s.t. } & C_{i}=\frac{1}{\ln 2}\left(u_{i}-v_{i}\right), \\
& \text { Polynomial approximation of } u_{i} \text { and } v_{i} \text { given by (6), } \\
& \text { Linear expressions for } x_{i} \text { and } y_{i} \text { (using (10)), } \\
& \text { Bounding constraints for } \Gamma_{j 1 k 1}, \Gamma_{j 1 k 2} \text { and } \Gamma_{j 2 k 2}(11), \\
& \operatorname{Tr}\left\{\Lambda_{i}\right\} \leq P_{\text {max }}, \operatorname{diag}\left\{\Lambda_{i}\right\} \succeq \mathbf{0}, 1 \leq i \leq N .
\end{array}
$$

The LPR essentially relaxes the feasible region of the original NL problem (3) and hence the optimal objective function value of the LP (12) serves as an UB to the optimal sum-rate. Since the LP is subject to the same power constraint as the original NL problem (3), the optimal solution to the LP is also a feasible solution to (3). In general, this feasible solution is not optimal to the original problem (3) and the corresponding objective function value of (3) yields a LB to the optimal sum-rate. After finding these bounds, BB strategy is employed to partition the search space so that both the largest UB and LB approach towards the global maximum as explained in Table I.

\section{NUMERICAL RESUlTS}

We compare the optimal sum-rate results with four special cases, viz., no interference sum-rate, optimal equal power allocation (OEPA), maximum equal power allocation (MEPA) and IA bound. In the no interference case, we assume that each user is isolated from all the other users. This is a hypothetical case in the current setup and is just meant to upper bound the optimal sum-rate. In OEPA, the total transmit power of each user is optimized with the assumption of equal power distribution over all Tx antennas [19]. In MEPA, there is no power control and each user transmits maximum power $P_{\max } / n_{t}$ over each Tx antenna. As mentioned earlier, IA 


$$
\begin{gathered}
\mathbf{S}_{i}=\left[\begin{array}{cc}
1+\sum_{j=1}^{N} \lambda_{j 1}\left(\tilde{h}_{j i}\right)_{11}\left(\tilde{h}_{j i}\right)_{11}^{\prime}+\lambda_{j 2}\left(\tilde{h}_{j i}\right)_{12}\left(\tilde{h}_{j i}\right)_{12}^{\prime} & \sum_{j=1}^{N} \lambda_{j 1}\left(\tilde{h}_{j i}\right)_{11}\left(\tilde{h}_{j i}\right)_{21}^{\prime}+\lambda_{j 2}\left(\tilde{h}_{j i}\right)_{12}\left(\tilde{h}_{j i}\right)_{22}^{\prime} \\
\sum_{j=1}^{N} \lambda_{j 1}\left(\tilde{h}_{j i}\right)_{21}\left(\tilde{h}_{j i}\right)_{11}^{\prime}+\lambda_{j 2}\left(\tilde{h}_{j i}\right)_{22}\left(\tilde{h}_{j i}\right)_{12}^{\prime} & 1+\sum_{j=1}^{N} \lambda_{j 1}\left(\tilde{h}_{j i}\right)_{21}\left(\tilde{h}_{j i}\right)_{21}^{\prime}+\lambda_{j 2}\left(\tilde{h}_{j i}\right)_{22}\left(\tilde{h}_{j i}\right)_{22}^{\prime}
\end{array}\right] \\
x_{i}=1+\sum_{j=1}^{N}\left(\left(\tilde{h}_{j i}\right)_{11}\left(\tilde{h}_{j i}\right)_{11}^{\prime}+\left(\tilde{h}_{j i}\right)_{21}\left(\tilde{h}_{j i}\right)_{21}^{\prime}\right) \lambda_{j 1}+\sum_{j=1}^{N}\left(\left(\tilde{h}_{j i}\right)_{12}\left(\tilde{h}_{j i}\right)_{12}^{\prime}+\left(\tilde{h}_{j i}\right)_{22}\left(\tilde{h}_{j i}\right)_{22}^{\prime}\right) \lambda_{j 2}+ \\
\sum_{j=1}^{N} \sum_{k=1}^{N}\left(\left(\tilde{h}_{j i}\right)_{11}\left(\tilde{h}_{j i}\right)_{11}^{\prime}\left(\tilde{h}_{k i}\right)_{21}\left(\tilde{h}_{k i}\right)_{21}^{\prime}-\left(\tilde{h}_{j i}\right)_{11}\left(\tilde{h}_{j i}\right)_{21}^{\prime}\left(\tilde{h}_{k i}\right)_{11}^{\prime}\left(\tilde{h}_{k i}\right)_{21}\right) \lambda_{j 1} \lambda_{k 1}+ \\
\sum_{j=1}^{N} \sum_{k=1}^{N}\left(\left(\tilde{h}_{j i}\right)_{12}\left(\tilde{h}_{j i}\right)_{12}^{\prime}\left(\tilde{h}_{k i}\right)_{22}\left(\tilde{h}_{k i}\right)_{22}^{\prime}-\left(\tilde{h}_{j i}\right)_{22}\left(\tilde{h}_{j i}\right)_{12}^{\prime}\left(\tilde{h}_{k i}\right)_{22}^{\prime}\left(\tilde{h}_{k i}\right)_{12}\right) \lambda_{j 2} \lambda_{k 2}+ \\
\sum_{j=1}^{N} \sum_{k=1}^{N}\left(\left(\tilde{h}_{j i}\right)_{11}\left(\tilde{h}_{j i}\right)_{11}^{\prime}\left(\tilde{h}_{k i}\right)_{22}\left(\tilde{h}_{k i}\right)_{22}^{\prime}+\left(\tilde{h}_{j i}\right)_{21}\left(\tilde{h}_{j i}\right)_{21}^{\prime}\left(\tilde{h}_{k i}\right)_{12}\left(\tilde{h}_{k i}\right)_{12}^{\prime}-\right. \\
\left.2 \Re\left(\left(\tilde{h}_{j i}\right)_{11}\left(\tilde{h}_{j i}\right)_{21}^{\prime}\right) \Re\left(\left(\tilde{h}_{j i}\right)_{12}\left(\tilde{h}_{j i}\right)_{22}^{\prime}\right)-2 \Im\left(\left(\tilde{h}_{j i}\right)_{11}\left(\tilde{h}_{j i}\right)_{21}^{\prime}\right) \Im\left(\left(\tilde{h}_{j i}\right)_{12}\left(\tilde{h}_{j i}\right)_{22}^{\prime}\right)\right) \lambda_{j 1} \lambda_{k 2}
\end{gathered}
$$

bound represents half the sum-rate achievable in the nointerference case. It should be noted that IA bound is considered just for the comparison purpose and the feasibility of actual IA over a limited number of signaling dimensions is still an open problem.

The channel model is assumed to be a combination of large scale and small scale fading components. On the large scale, we assume that channel suffers from an exponential path loss with a path loss factor of 3 and from log-normal shadowing with a standard deviation of $1 \mathrm{~dB}$. Small scale fading effects are modeled as Rayleigh distributed. Antennas at both the Tx and $\mathrm{Rx}$ of all the users are assumed to be independent in terms of small-scale fading but perfectly correlated in terms of lognormal shadowing. The interference power is controlled by varying the $M U I$ factor (high $M U I$ means high interference power).

Fig. 4 presents a comparison of the optimal sum-rates of the current IC with the four special cases in both the low $(M U I=1)$ and the high $(M U I=5)$ interference scenarios $\left(n_{t}=n_{r}=2\right)$. It is evident that the sum-rates achievable in MEPA are significantly lower than the optimal sum-rates and the ones achievable in OEPA, with the difference being more prominent in the high interference scenario. This highlights the importance of optimal power control over spatial dimension in the MIMO ICs. As expected, the optimal sum-rate is better than OEPA in both the interference scenarios. This highlights the importance of optimizing power over each spatial channel rather than just optimizing the total transmit power and distributing it equally over all the channels.

We also compare the optimal power control sum-rate with the IA bound. This comparison suggests that the optimal sum-rate is significantly higher than the IA bound in both the interference scenarios considered. This is an interesting result since it shows that even a simple power control scheme can achieve better sum-rate than IA bound in low and even moderately high interference. This is consistent with the facts that treating interference as noise is optimal and IA bound is sub-optimal in the low interference regime. It is also noted in Fig. 4 that the rate of decrease of per user sum-rate is much less for $N>M U I$. This is due to the fact that when we increase the value of $N$, the net interference experienced by an Rx node goes on increasing until the value of $N$ reaches $M U I$, after which the expected interference almost becomes constant. On the other hand, IA bound implicitly assumes
$M U I=N$ (all users strongly interfere with each other). To have a fair comparison, we set $M U I=N$ in our system and present the results in Fig. 5. As expected, the optimal sum-rate is higher than the IA bound for a reasonable number of users. However, it gets worse than IA bound when the interference power becomes very high. This is due to the fact that a higher fraction of users is turned off as we move from the low to high interference regimes. In particular, we note that the importance of interference avoidance by turning off the users and/or limiting the transmission over a single spatial channel increases as we move from low to high interference regime. In the limiting case of very high interference, it is optimal most of the time to let only $n_{t}$ (assuming $n_{t}=n_{r}$ ) users transmit by confining their power over their respective strongest channels (beamforming). The detailed study of the optimal power allocation strategies is out of the scope of this publications and will be presented soon in a separate publication.

In Fig. 6, we study the effect of $S N R_{\min }$ on the optimal sum-rate. Since $S N R_{\min }$ defines the noise power, it indirectly affects the relative strength of interference as compared to noise. In particular, low values of $S N R_{\min }$ corresponds to a low interference scenario due to the lower relative strength of interference as compared to noise (and vice-versa). From the results we note that the optimal power control performs better in the low interference scenario and IA bound performs better in the high interference scenario. The reason for this is again the optimality of turning off a higher fraction of users in the high interference regime as compared to the low interference regime.

\section{Conclusions}

In this paper, we have solved the long-standing open problem of finding the optimal sum-rate of a peer-to-peer MIMO IC treating interference as Gaussian noise. We have assumed that all the users share a single frequency band and no precoding is performed over time. This problem is essentially reduced to the problem of finding the optimal power allocation over different spatial channels. The importance of optimal power control over spatial channels is highlighted by comparing the optimal results with two sub-optimal cases, viz., MEPA and OEPA. We have also compared the optimal results with the sum-rate achievable by IA and shown that the optimal power allocation strategy achieves higher sum-rates than IA 


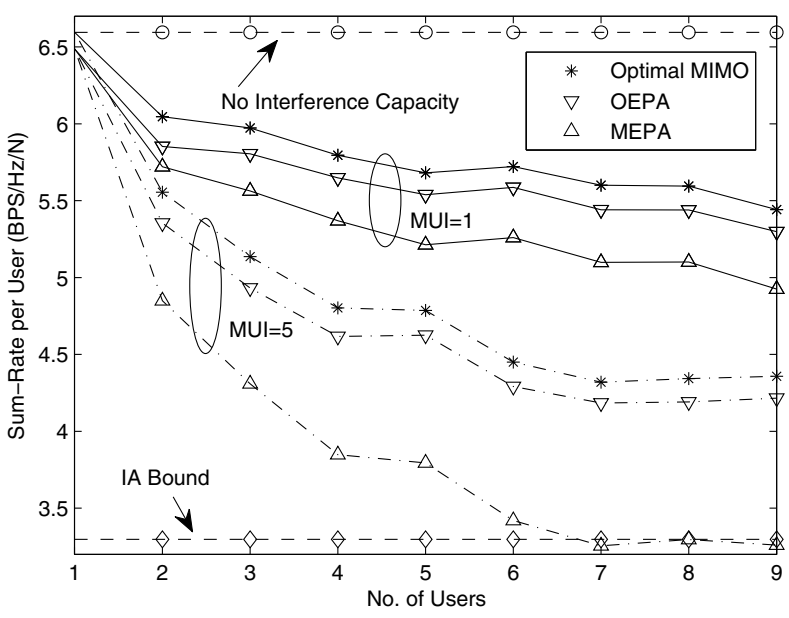

Fig. 4. Comparison of optimal sum-rate with various sub-optimal MIMO techniques in high $(M U I=5)$ and low $(M U I=1)$ interference scenarios $\left(S N R_{\text {min }}=1 \mathrm{~dB}\right)$.

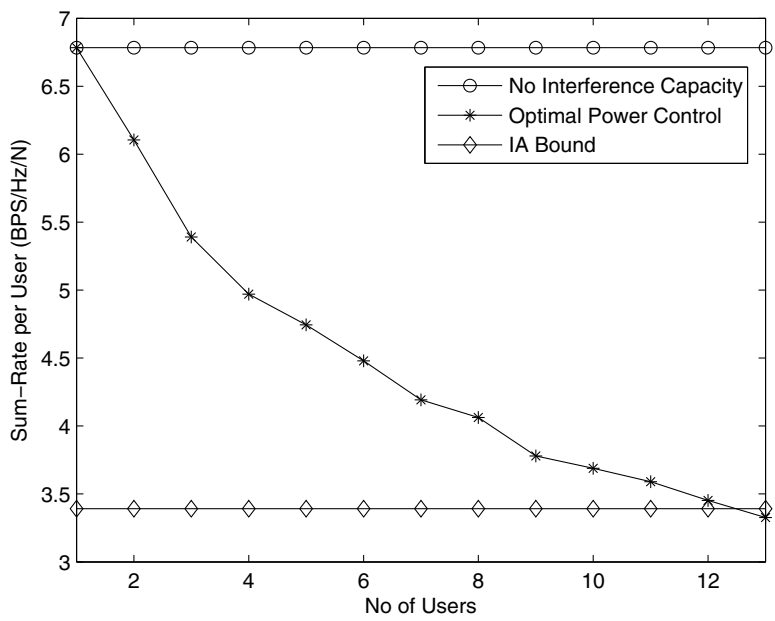

Fig. 5. Comparison of optimal sum-rate with no-interference capacity and IA bound for the case when $M U I=N\left(S N R_{\min }=1 \mathrm{~dB}\right)$.

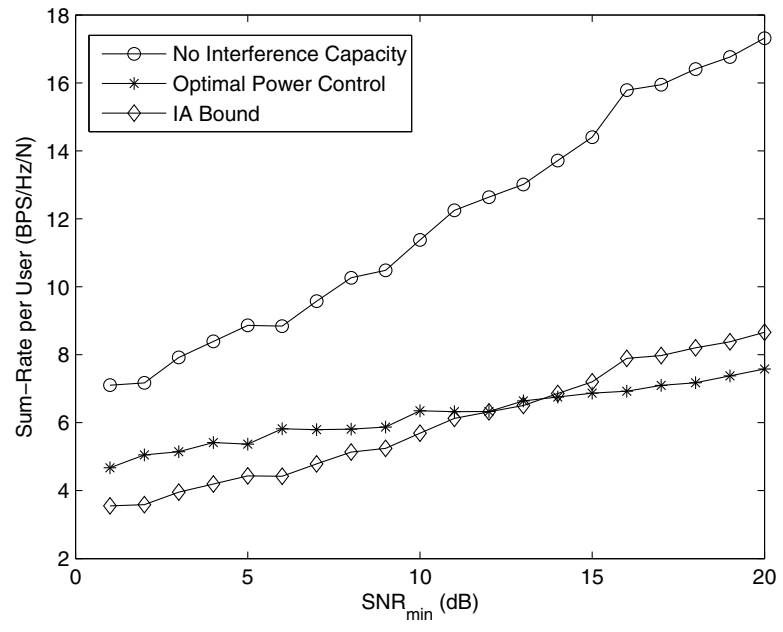

Fig. 6. Comparison of optimal sum-rate with no-interference capacity and IA bound for various values of $S N R_{\min }(M U I=5, N=4)$. in low and even moderately high interference scenarios. On the other hand, the importance of doing explicit IA in high interference scenarios is also highlighted by the results.

\section{ACKNOWLEDGMENTS}

We would like to thank Qualcomm Inc. for sponsoring this research. We would like to thank Mr. Justin Kelly for his insightful inputs in developing the system model. Thanks are due to Dr. Thomas Hou and Kevin Liu for their help in understanding $\mathrm{BB} / \mathrm{RLT}$ algorithm.

\section{REFERENCES}

[1] C. E. Shannon, "Two-Way Communication Channels", in Proc. $4^{\text {th }}$ Berkeley Symp. Math. Stat. Prob., Berkeley, CA, 1961, pp. 611-644.

[2] R. Ahlswede, "The Capacity Region of a Channel with Two Senders and Two Receivers", Annals Probability, vol. 2, no. 5, pp. 805-814, 1974.

[3] A. B. Carleial, "Interference Channels", IEEE Trans. Inform. Theory, vol. IT-24, pp. 60-70, Jan. 1978.

[4] H. Sato, "Two-User Communication Channels", IEEE Trans. Inform. Theory, vol. IT-23, pp. 295-304, May 1977.

[5] A. B. Carleial, "A Case where Intereference does not Reduce Capacity", IEEE Trans. Inform. Theory, vol. IT-21, pp. 569-570, Sept. 1975.

[6] T. S. Han and K. Kobayashi, "A New Achievable Rate Region for the Interference Channel", IEEE Trans. Inform Theory, vol. 27, no. 1, pp. 49-60, Jan. 1981

[7] R. Etkin, "Spectrum sharing: Fundamental Limits, Scaling Laws, and Self Enforcing Protocols", Ph.D. dissertation, Univ. of California, Berkeley, Dec. 2006

[8] R. Etkin, D. Tse and H. Wang, "Gaussian Interference Channel Capacity to Within One Bit", IEEE Trans. Inform. Theory, vol. 54, no. 12, pp. 5534-5562, 2008.

[9] M. Maddah-Ali, A. Motahari and A. Khandani, "Signaling over MIMO Multi-Base systems- Combination of Multi-Access and Broadcast Schemes", in Proc. IEEE Int. Symp. Inform. Theory, Seattle, WA, 2006, pp. 2104-2108.

[10] V. R. Cadambe and S. A. Jafar, "Interference Alignment and the Degrees of freedom for the $K$ user interference channel", IEEE Trans. Inform. Theory, vol. IT-54, no. 8, pp. 3425-3441, Aug. 2008.

[11] V. R. Cadambe, S. A. Jafar, and S. Shamai (Shitz), "Interference Alignment on the Deterministic Channel and application to Fully Connected AWGN Interference Networks", IEEE Trans. Inf. Theory, vol. IT-55, no. 1, pp. 269-274, Jan. 2009.

[12] M. Shen, A. Høst-Madsen and J. Vidal, "An Improved Interference Alignment Scheme for Frequency Selective Channels", in Proc. IEEE Int. Symp. on Inform. Theory (ISIT), July 2008, pp. 559-563.

[13] S. W. Choi, S. A. Jafar and S.-Y. Chung, "On the Beamforming Design for Efficient Interference Alignment", IEEE Comm. Lett., vol. 13, no. 11 , pp. 847-849, 2009.

[14] T. Gou and S. A. Jafar, "Degrees of Freedom of the K User MIMO Interference Channel", in Proc. Asilomar Conf. Sig., Sys. and Computers, Pacific Grove, CA, 2008, pp. 126-130.

[15] R. Tresch, M. Guillaud and E. Riegler, "On the Achievability of Interference Alignment in the K-user Constant MIMO Interference Channel", in Proc. IEEE Workshop on Statistical Signal Processing, Cardiff, U.K., Sept. 2009, pp. 277-280.

[16] B. S. Blum, "MIMO Capacity with Interference", IEEE J. Select. Areas Commun., vol. 21, pp. 793-801, June 2003.

[17] S. Ye and R. S. Blum, "Optimized Signaling for MIMO Interference Systems with Feedback", IEEE Trans. Signal Process., vol. 51, no. 11, pp. 2839-2848, Nov. 2003

[18] W. Yu, W. Rhee, S. Boyd, and J. M. Cioffi, "Iterative Water-Filling for Gaussian Vector Multiple-Access Channels", IEEE Trans. Inform. Theory, vol. 50, no. 1, pp. 145-152, Jan. 2004.

[19] J. Liu, Y. T. Hou, Y. Shi and H. Sherali, "On the Capacity of Multiuser MIMO Networks with Interference", IEEE Trans. Wireless Commun., vol. 7, no. 2, pp. 488-494, Feb. 2008.

[20] H. D. Sherali and W. P. Adams, A Reformulation-LinearizationTechnique for Solving Discrete and Continuous Nonconvex Problems. Boston, MA: Kluwer Academic Publishing, 1999.

[21] S. W. Peters and R. W. Heath Jr., "Cooperative Algorithms for MIMO Interference Channels", [Online], Feb. 2010, Available: http://arxiv.org/PS_cache/arxiv/pdf/1002/1002.0424v1.pdf. 